\title{
Location, location, location: Nuclear structure regulates gene expression in neurons
}

\author{
Emily Brookes and Antonella Riccio \\ MRC Laboratory of Molecular Cell Biology, University College London, \\ Gower Street, London UK WC1E 6BT \\ Corresponding email: a.riccio@ucl.ac.uk
}

\begin{abstract}
Genome architecture plays a critical role in regulating the expression of genes that are essential for nervous system development. During neuronal differentiation, spatially and temporally regulated transcription allows neuronal migration, the growth of dendrites and axons, and at later stages, synaptic formation and the establishment of neuronal circuitry. Genome topology and relocation of gene loci within the nucleus are now regarded as key factors that contribute to transcriptional regulation. Here, we review recent work supporting the hypothesis that the dynamic organization of chromatin within the nucleus impacts gene activation in response to extrinsic signalling and during neuronal differentiation. The consequences of disruption of the genome architecture on neuronal health will be also discussed.
\end{abstract}

\section{Highlights}

- Nuclear architecture is essential for transcriptional activation in neurons

- Changes in chromosome contacts occur during neuronal differentiation

- Proteins that organise genome loops include cell type specific transcription factors

- Alteration of genome topology is associated with neurological disorders 


\section{Introduction}

Development and function of the mammalian central nervous system (CNS) poses a great challenge for gene regulation. A fairly homogenous population of neuronal precursors gives rise to a dazzling array of cell types that include neurons, oligodendrocytes, astrocytes, microglia and endothelial cells [1]. Moreover, single cell sequencing experiments have revealed an increasing number of neuronal types with molecularly distinct features [2]. This multitude of neuronal and non-neuronal cell types is generated through careful control of cell proliferation, migration, and terminal differentiation. The CNS largely retains plasticity throughout the life of the organism and this ability to adapt to the external environment is necessary for learning, and ultimately survival.

In all eukaryotic cells, the genome is heavily compacted to fit inside the nucleus. Despite this compression, genes must remain accessible for transcription, DNA replication and other nuclear processes. In the nucleus, chromatin is highly organised within the three-dimensional (3D) space, allowing tight control of gene expression. Genome conformation is regulated at multiple levels, from the wrapping of DNA around histones to form nucleosomes, to epigenetic modifications of DNA and histones, and ATP-dependent histone sliding and exchange. In addition, a key role is played by the formation of higher order domains, and by the movement of genomic regions in relation to nuclear landmarks. Interplay within and between these levels of regulation allows precise gene regulation.

In this review, we will describe recent work demonstrating how genome topology and nuclear positioning influence gene transcription in neurons. We will illustrate the unforgiving nature of nuclear structure deregulation by describing neurological diseases resulting from mutations affecting genome architecture.

\section{The nuclear lamina is a repressive compartment for transcription}

The radial arrangement of the genome within the nucleus is highly ordered. The nuclear periphery is enriched in heterochromatin and is generally associated with gene-poor or transcriptionally silent regions of the genome; it is therefore considered a compartment that represses transcription. During neuronal development, certain genes move away from the lamina towards the nuclear interior, and this event is concomitant with transcriptional activation [3-5] (Figure 1A). Similarly, in the adult brain, $B d n f$ activation by kainate-induced seizures is accompanied by movement of the Bdnf gene away from the nuclear periphery [6]. Such movement may relocate genes away from a repressive environment, or towards sites rich in transcriptional apparatus. In 
embryonic cortical neurons for example, activity-dependent genes such as cFos and Gadd45b relocate to RNA polymerase II (RNAPII) foci in response to depolarization $[7,8]$

Lamina-associated domains (LADs) are large genomic regions that localize at the nuclear lamina and guide the spatial organization of the interphase nucleus [9]. LADs are predominantly gene-poor and contain silent genes bearing repressive histone modifications. Detailed mapping of LADs in mouse embryonic stem cells (ESCs), neuronal progenitor cells (NPCs) and astrocytes revealed that in most cases, dissociation from the nuclear lamina protein LaminB1 resulted in the transcription of genes involved in neuronal and astrocyte specification [5]. Some LADs are constitutive, but hundreds of genes change their lamina interaction during differentiation. Importantly, not only have correlations between radial location and gene expression been observed in a variety of cell types but tethering of genes to the lamina was found to induce gene silencing [10]. Conversely, forced activation of endogenous genes within a LAD was sufficient to relocate the region to the nuclear interior [11]. Interestingly, during the early stages of neuronal differentiation, a substantial group of genes detached from the lamina but remained inactive [5]. These findings suggest that while movement away from the lamina is often necessary for triggering transcription, it is not capable of inducing gene activation per se (Figure 1A). Nonetheless, these genes were more prone to transcriptional activation at later differentiation stages [5], indicating that release from the lamina may prime them for rapid expression when additional extrinsic signals come into play. This is in accordance with data showing that decondensation of endogenous genes within a LAD was sufficient to relocate the region to the nuclear interior, without affecting transcription [11].

A striking example of how nuclear geometry can be regulated in the nervous system is provided by the dramatic folding of the nuclear membrane observed in hippocampal neurons [12]. The nuclear infoldings were dynamic, and increased in number following synaptic activation, leading to an increase in surface area of the transcriptionally repressive lamina. These nuclear membrane invaginations may facilitate the relay of calcium signals to the nucleus by generating smaller functional compartments and may affect transcriptional regulation. Quiescent neural stem cells also develop nuclear envelope invaginations, which may contain telomeres and other heterochromatin domains [13]. Although it is unclear whether these invaginations are functionally related to the infoldings described in neurons, they also affect the surface area of a transcriptionally repressive compartment and may lead to the existence of signaling microdomains.

Role of the nuclear lamina in the pathogenesis of neurological disorders 
The protein components of the lamina are critical for its regulatory function (Box 1), and disruption of them may cause neurological diseases. LaminB1 is required for brain development $[14,15]$, for dendritogenesis in cortical neurons [16], and for the regulation of gene expression during olfactory neuron differentiation [17]. Mice lacking the LaminB1 paralog, LaminB2, exhibit cortical development defects $[14,15]$. Importantly, loss of function mutations of $L M N B 1$ in humans result in neural tube defects [18], whereas mutations of the LMNA gene gives rise to a group of disorders known as the laminopathies [19]. In addition to the well-known premature ageing disease Hutchinson-Gilford progeria syndrome, laminopathies include more than 15 distinct diseases characterised by muscular, metabolic and neurological symptoms. Although the mechanisms are undoubtedly complex and remain to be fully elucidated, genes and indeed entire chromosomes have been shown to change radial nuclear position, implying that transcriptional regulation is likely an important facet of the disease (Figure 3A).

\section{Chromosomes are organised into topologically associating domains (TADs)}

The genome is arranged in self-interacting genomic regions known as topologically associating domains (TADs) $[20,21]$. DNA sequences within a TAD interact more frequently with each other than with regions located outside. TAD boundaries function as transcriptional insulators. Chromosome conformation capture technologies, which use ligation to link regions that are close together in 3D space, have allowed identification of genomic contacts on a large scale. Importantly, the existence of TADs was recently confirmed genome-wide using a ligation-independent method that relies instead on genome amplification and DNA sequencing from nuclei dissected into ultrathin sections [22]. TADs have been identified in all cell types studied, including mouse and human brain $[23 \bullet \bullet, 24 \bullet]$. TAD organisation is generally stable, and some TAD boundaries are conserved across differentiation stages, cell types and even between species [21]. Multiple lines of evidence indicate that TADs are formed by loading of cohesin onto the genome, which uses energy derived from ATP hydrolysis to spool the DNA through its ring-like structure, forming a TAD [25] (Figure 2). Extrusion stops when cohesin encounters CTCF (CCCTC-binding factor) bound to the DNA, resulting in the majority of TAD boundaries containing both CTCF and cohesin.

A recent study performed ultradeep mapping of genome interactions in mouse ESCs, NPCs and cortical neurons (CNs) differentiated in vitro, as well as NPCs and CNs purified from embryonic cortex $[23 \bullet \bullet]$. The authors found that during neuronal differentiation, a global reorganization of chromatin takes place (Figure 1B). Although many TAD boundaries remain unchanged, in general TADs become fewer and larger, average intra-TAD contacts become stronger and average inter-TAD 
contacts are depleted. Importantly, formation of new TAD boundaries was observed close to developmentally regulated genes as they became transcriptionally activated during neuronal differentiation. However, forced activation of a silent genomic region was insufficient to create a TAD boundary. This suggests that transcription alone is not sufficient to induce the structural changes associated with boundary formation, but rather the formation of new TAD boundaries during neuronal development may be permissive or instructive for the expression of specific genes.

\section{Regulation of TAD organisation during neuronal development}

Bonev et al., showed three distinct classes of TAD boundaries in NPCs and CNs: first, boundaries characterized by the presence of CCCTC-binding factor (CTCF) and cohesin; second, CTCF-negative, cohesin-positive boundaries that are close to active gene promoters; and finally, boundaries without active marks of transcription that are enriched for repeat elements [23••]. Below we will discuss the major players in TAD regulation and their role in neuronal gene regulation.

1. CTCF. CTCF is a DNA-binding protein that plays a critical role in determining looping interactions at various levels of the architectural hierarchy [26]. CTCF can regulate both inter- and intra-chromosomal interactions and is integral in establishing TAD boundaries and sub-TAD looping. A recent study used an acute depletion system to remove CTCF from murine ESCS, NPCs and astrocytes [27••]. Dramatic loss of looping and insulation at TAD boundaries was observed upon CTCF depletion, which caused the merging of neighboring TADs and the formation of ectopic contacts across the original TAD borders. These changes were coincident with transcriptional dysregulation, although the defects were less severe in astrocytes than ESCs or NPCs, suggesting a less prominent role for CTCF in terminally differentiated cells. This is in keeping with an increasing proportion of CTCF-negative borders during neuronal differentiation [23••]. Interestingly, TAD insulation was re-established upon restoration of CTCF levels in ESCS and NPCs, but not in resting astrocytes, indicating that passage through the cell cycle may be indispensable for restoring TAD boundaries $[27 \bullet \bullet]$.

In mice, increasing evidence indicates that CTCF influences the expression of neuronal genes [2830], including the protocadherin genes, whose stochastic variation in expression levels generates neuronal diversity $[28,29 \bullet]$. In the developing cortex, CTCF regulates NPC differentiation and survival [31], and at later stages, dendritic arborization and spine development [28]. In cortical interneurons, CTCF regulates the expression of genes involved in fate determination, and mutations of the CTCF gene lead to defects in cell identity and neuronal migration [30]. Loss of CTCF in the hippocampus is associated with impaired long-term potentiation, reduced dendritic spine density, 
and defects in spatial and fear memory [29•]. As well as finding many deregulated genes in the hippocampus of CTCF-knockout mice, including protocadherin and learning-related genes, the authors found a significant lack of upregulation of Bdnf and $\operatorname{Arc}$ genes in response to fear conditioning. The absence of induced Bdnf and Arc expression was associated with disruption of the genome architecture surrounding the gene loci. This suggests that stimuli-responsive gene expression is affected by CTCF-regulated genome architecture, as well as developmentallyregulated gene programs.

2. Cohesin. Cohesin is a ring-like multiprotein complex initially described as part of the complex that holds sister chromatids together during anaphase and was later found to have an additional role in genome architecture [32]. Cohesin is critical for genome looping in neural stem cells differentiated into astrocytes in vitro [33]. Loss of cohesin causes loss of TAD insulation, increasing inter-TAD interactions and resulting in widespread transcriptional dysregulation [33]. The transcriptional changes resulting from loss of cohesin may cause a number of downstream effects, depending on the cell type. The nervous system may be particularly sensitive to cohesin-mediated transcription changes as mutation of cohesin in mice induces behavioral defects, possibly due to transcriptional dysregulation of genes necessary for dendrite and synapse development $[34,35]$. Importantly, mutations of the cohesin pathway in humans cause Cornelia de Lange syndrome, a severe developmental disorder that manifests with neurological symptoms, including psychomotor delay and intellectual disability [36].

3. Topoisomerase IIß (TOP2B). TOP2B is a member of the DNA topoisomerase family responsible for relaxing the DNA supercoils that change DNA topology, and often co-occupies TAD boundaries with CTCF and cohesin [37]. An early study indicated that TOP2B plays an essential role in neuronal development, as deletion of the gene in mice induced profound defects in motor and sensory neuron innervation [38]. In cortical neurons, TOP2B and CTCF are found in a complex bound to many neuronal genes, in keeping with the idea that TOP2B may regulate genome structure in the brain [39] and that this may explain some of the effects of TOP2B loss on neuronal development. An intriguing finding of this study is that TOP2B promotes expression of activity regulated genes, at least in part, by causing DNA double strand breaks in response to neuronal activity, suggesting that relieving topological constraint may be critical for gene expression.

\section{Diseases caused by mutations in TAD boundaries}

TAD boundaries insulate genomic regions from each other, and therefore perturbation of TAD boundaries leads to inappropriate interactions between chromatin regions, including aberrant 
enhancer-promoter looping (Figure 3B). Recent technological advances have allowed sequencing of patient samples to go beyond exome sequencing and identify more variants in the non-coding portion of the genome. As a result, it is increasingly recognized that mutations in TAD borders contribute significantly to both Mendelian heritable disorders and complex multifactorial diseases.

One example is provided by Fragile $X$ syndrome, a disease marked by severe cognitive defects that is caused by a repeat expansion in the FMR1 gene. FMR1 encodes Fragile X Mental Retardation Protein (FMRP), a translational repressor essential for normal cognitive development [40]. An interesting recent study indicated that the repeat expansion within FMR1 interferes with CTCF binding sites, disrupting a TAD border and inhibiting FMR1 expression [41•]. The authors hypothesize that TAD disruption alters looping of the FMR1 promoter to enhancers. Importantly, this disease mechanism may extend to other neurological disorders, as the authors found a striking correlation between disease-associated DNA repeats and TAD boundaries, at boundaries with high CpG island density [41•]. A case study of the rare neurological disorder Autosomal Dominant adultonset demyelinating LeukoDystrophy (ADLD) provides another example of how nuclear architecture may influence gene regulation [42]. The deletion observed in ADLD patients eliminates a TAD boundary, allowing an enhancer to contact the LMNB1 gene, amplifying its expression. LMNB1 encodes the LaminB1 protein that is an integral part of the nuclear lamina, itself a repressive compartment for transcription (Box 1). Therefore, this example highlights two points at which gene regulation through nuclear architecture can be manipulated in disease. LMNB1 overexpression causes progressive CNS demyelination, autonomic dysfunction, ataxia and cognitive impairment.

A survey of 922 deletion sites found in the DECIPHER clinical genome database further revealed that many disease-associated deletions overlap TAD boundaries [43]. Analysis of enhancer usage and gene functions suggested that part of the disease phenotypes could be attributed to the adoption of new enhancers following boundary disruption, and consequent transcriptional changes. The phenotype of the disorders in this survey is complex and often involves several organs, however intellectual disability, along with congenital abnormalities, is one of the most common symptoms [43]. This suggests that the developing brain may be more sensitive to epigenetic insults, compared to other tissues. In one interesting example from this study, deletion of a boundary brings an ectopic foetal brain enhancer into the regulatory domain of FOXG1 [43]. FOXG1 encodes a transcription factor that is expressed in the developing nervous system from the earliest stages and is critical for regulating multiple important processes including dorso-ventral patterning, neural precursor proliferation, neuronal differentiation, and neuronal cell fate [44]. Patients with the boundary deletion exhibit neurodevelopmental defects, with intellectual disability, developmental 
delay, and postnatal microcephaly [43], similar to that caused by mutations in FOXG1 itself [45]. However, a recent study suggests that the deletion causes loss of FOXG1 regulatory elements that are of more significance in the patient phenotype than the TAD boundary deletion [46].

\section{Dynamic looping generates sub-TADs}

Within the TADs themselves, highly variable restructuring of the chromatin architecture creates sub-TAD loops, which may link genes to their enhancers or to other co-regulated genes (Figure 2). Sub-TAD looping is tightly controlled to ensure correct spatiotemporal gene regulation, and varies markedly between cell types and developmental stages. Although CTCF is implicated in the formation and maintenance of sub-TADs, other mechanisms are also involved.

During neuronal differentiation, average intra-TAD contacts become stronger [23••]. Interactions independent of CTCF binding were detected between promoters of active genes at all developmental stages and correlated with gene expression. Surprisingly, the authors identified very long-range $(>30 \mathrm{MB}$ ) looping events between activated genes in ESCs, NPCs and CNs. These interactions span TADs, and even occur between genes on different chromosomes. Contact enrichment correlated with the number of exons, RNA splicing events and gene transcription levels. Contacts between transcribed genes have also been described in astrocytes, where the astrocytespecific gene Gfap has been found to associate with a number of other expressed genes [47]. One recent exciting discovery was that during olfactory neuron differentiation, olfactory receptor genes aggregate with each other and with their enhancers [48••]. In progenitor cells this cluster seems to be repressive for transcription. However, in mature olfactory sensory neurons, the transcribed olfactory receptor gene separates from the repressive cluster and forms a separate hub together with its enhancers. Thus, long range trans interactions create both an activating structure for a single gene, and a repressive structure to prevent expression of more than one olfactory receptor gene per cell $[48 \bullet \bullet]$.

At a finer level, interactions were observed between the promoter and the entire transcribed region of expressed genes, as well as the termination site [23••] (Figure 2 ). Such intra-gene contacts may be required for RNA splicing and RNAPII recycling. Looping events that allowed the establishment of enhancer-promoter contacts were primarily identified within TADs and, as for contacts between promoters, were predominantly independent of CTCF binding. As perhaps expected, enhancer-promoter contacts correlated with transcriptional activation, further indicating the impact of highly dynamic changes of chromatin architecture on gene expression during neuronal differentiation. 


\section{Regulation of sub-TAD looping during neuronal development}

Proteins implicated in establishing or stabilizing enhancer-promoter loops include CTCF, YY1 [49,50•], RNAPII [51], HNRNPU [52], the chromatin remodeler SMARCA4 [53,54], and the Mediator complex [55]. Neural-specific transcription factors have also recently been implicated in organizing genome topology $[23 \bullet \bullet, 48 \bullet \bullet$. Although CTCF does regulate sub-TAD looping, its role diminishes during neuronal differentiation, concomitant with a decrease in the number of CTCF binding sites across the genome [50•]. Similarly, in the adult cortex, cerebellum and olfactory bulb, fewer CTCF binding sites were found when compared to the embryonic brain. Decreased CTCF binding correlated with lower levels of CTCF expression $[29 \bullet, 50 \bullet]$, which also varied between cell types within the CNS [29•]. Interestingly, the developmental decrease of global CTCF and of CTCF binding was uniquely observed in the neural lineage [50•]. ESCs and NPCs showed a similar number of looping events despite reduced CTCF occupancy in NPCs, demonstrating that a higher percentage of NPC-only loops were not anchored by CTCF [50•]. This indicates that other proteins contribute to maintaining chromatin architecture in differentiated neurons.

Ying Yang 1 (YY1) is a zinc finger protein that is strongly enriched at interaction sites between NPC-specific genes and enhancers [50•]. YY1 is critical for neuronal differentiation [56] and in humans, haploinsufficiency of YY1 results in loss of H3K27ac at enhancers, transcriptional dysregulation and intellectual disability [57]. Knockdown of YY1 in NPCs reduced the interaction frequency between the YY1-bound genes Sox2, KIf4 and Zfp462 and their enhancers, concomitantly inhibiting their expression levels [50•]. Interestingly, another study has suggested that YY1 regulates enhancer-promoter looping not only in NPCs but also in pluripotent cells and in other differentiated cell types [49].

The lineage-specific factors that help to organize genome structure also undergo substantial changes during neuronal differentiation (Figure 1C). In ESCs, contacts occur between sites bound by Polycomb, and sites bound by pluripotency-specific transcription factors, such as Nanog. During neuronal differentiation, expression levels, chromatin occupancy and the contacts mediated by these factors all decrease. In NPCs, contacts occur between regions bound by the transcription factor Pax6 [23••], which is a master regulator of the NPC state. NeuroD2 and Tbr1 were also found at contact sites during neuronal development, with interactions increasing during differentiation to peak in mature CNs. Taken together, these findings indicate that dynamic organization of chromatin contacts around developmental-stage specific transcription factors within TAD boundaries significantly affects gene expression. Similarly, in olfactory neurons, the transcription factor Lhx2 
and its cofactor Ldb1 were necessary for the interaction of olfactory receptor genes with each other and with enhancers $[48 \bullet \bullet]$.

\section{Consequences of alterations in enhancer-promoter looping}

Disruption of enhancer-promoter loops (Figure 3 C) has been linked to a number of neurological diseases. For example, single nucleotide polymorphisms (SNPs) have been found at sites that physically interact with genes implicated in schizophrenia $[24 \bullet, 58]$. SNPs that may disrupt chromatin looping have been identified at contact sites of the risk locus CACNA1C [58], the antipsychotic drug target gene DRD2, several acetylcholine receptors, and genes involved in excitatory synaptic transmission [24•]. Although it is not clear whether all of these mutations interfere with expression of their partnered schizophrenia-related gene, at least in the case of FOXG1 a mutation in a region found to loop to the promoter abrogated FOXG1 expression [24•]. Similarly, SNPS associated with four neurodegenerative disorders (Frontotemporal lobar degeneration, Alzheimer's disease, Parkinson's disease and Amyotrophic lateral sclerosis) significantly overlap with CTCF binding sites [59]. SNPs at interaction sites can stimulate inappropriate expression as well as abrogate expression. One SNP associated with Frontotemporal lobar degeneration was found to promote CTCF-mediated looping from cis-regulatory elements to the TMEM106B promoter, increasing its expression [59]. Disrupted looping in the absence of specific mutations has also been found in neurological disease, for example at the GAD1 locus, which encodes the enzyme that synthesises the neurotransmitter GABA, in schizophrenia [60].

Finally, it is tempting to speculate that changes in genomic architecture could represent an early pathogenic event associated with predisposition for neurological diseases, thereby implicating that neuropsychological disorders that manifest later in life may be rooted into genome conformational changes that occurred at earlier developmental stages.

\section{Conclusions}

It is becoming increasingly clear that genome topology and radial nuclear location affect genome organization and spatiotemporal activation of gene expression in the nervous system. There are many factors that can influence transcription, including epigenetic modifications of DNA and histones, nucleosome remodeling, non-coding RNAs and region relocation to specific nuclear compartments. Many of these mechanisms intercalate with genome topology and exert changes through it. Folding of the genome into TAD and sub-TAD compartments plays a key role in the regulation of gene expression as this, among other events, limits the contacts between genes and 
their enhancers and co-regulated genes. Of note, genome 'contacts' are highly dynamic events that indicate proximity of sequences in 3D space and frequency of interaction, rather than fixed structures. Importantly, radial location of genes within the nuclear space affects both the chromatin environment and promoter accessibility to other genes and regulatory regions. Many neurological disorders can arise as a direct result of dysregulation of genome architecture, both through mutations of genes encoding proteins that have a crucial role in establishing or maintaining appropriate chromatin structure, or through mutations in the DNA sequences at critical contact points (Figure 3). Importantly, changes in genome topology may confer transcriptional priming, rather than activation, and potentially provide the biological basis for predisposition to neuropsychiatric disorders. Although the contribution of genome architecture to the pathogenesis of neurodevelopmental disorders awaits further investigation, technologies aimed at modifying chromatin structure may help restore appropriate gene expression in diseased neurons.

\section{Acknowledgments}

We thank Hamish Crerar and Catia Andreassi for reading the manuscript and providing useful suggestions. A.R. is a recipient of a Wellcome Trust Investigator Award and E.B. is supported by a Marie Skłodowska-Curie Postdoctoral Fellowship. The authors declare no conflicts of interest.

\section{Box 1 - Constituents of the lamina}

The major structural components of the nuclear lamina are the A-type lamins $A$ and $C$ that are alternatively spliced from the LMNA gene, and the B-type lamins B1 and B2. Expression of LaminB1 and the laminB receptor (LBR) decreases during neuronal development [23••]. Interestingly, lamins are not required for the interaction of LADs with the nuclear envelope but are necessary for tethering heterochromatin at the periphery [61]. The lamina is punctuated by nuclear pore complexes, large structures that mediate molecular trafficking between the nucleus and cytoplasm [62]. Although lamina association is generally negative for transcription, this is not always the case for nuclear pores. In NPCs, the transcription factor Sox2 and Nucleoporin153 (Nup153) bind to shared target genes [63], providing a striking example of how interaction of a lineage-specific transcription factor with the nuclear pore complex may regulate gene expression. The interaction between Nup153 and Sox2 is essential for the maintenance of NPCs, and Nup153 expression declined when NPCs differentiated into neurons and astrocytes. Interestingly, while the interaction of Nup153 with gene promoters correlated with transcriptional activation, binding at gene ends 
resulted in repression, suggesting that nuclear pores play a complex role in regulating transcriptional output.

\section{Figure legends}

Figure 1. Neuronal differentiation is marked by genome architecture changes

There are marked differences at all hierarchical levels of genome architecture between pluripotent embryonic stem cells (ESCs), multipotent neural progenitor cells (NPCs) and terminally differentiated cortical neurons (CNs). A. Following activation, many neuronal genes move radially toward the centre and away from the repressive nuclear periphery. It should be noted that dissociation from the lamina often reflects the acquisition of transcriptional competence rather than gene activation. B. At TAD level, changes in the boundaries of some TADs, depicted as triangles, lead to the formation of neural specific boundaries. Increasing colour intensity represents regions that show more frequent interaction. C. At the finest level, dynamic changes in sub-TAD looping and cell type specific factors induce local loop formation.

\section{Figure 2. Sub-TAD looping events}

Intra-TAD contacts are dynamic and cell type specific. Active genes show intra-gene interactions between promoter, coding region and 3' end, which may regulate splicing or RNAPII recycling. Loops are also formed between active promoters and their cell type-specific enhancers. Finally, interactions may occur between different active, but not inactive, genes, and correlate with the levels of gene expression.

\section{Figure 3. Mechanisms of disease caused by alteration of genome topology}

Disruption of chromosome organization is associated with the transcriptional dysregulation observed in many neurological disorders. A. 'Organiser' genes encode proteins with roles in configuring the genome within the 3D nucleus. Mutation in 'organiser' genes causes loss of these important proteins, and thereby dramatic downstream effects on gene expression. Examples include mutations in cohesin, YY1, CTCF, and Lamins A and B1. B. Mutations in TAD boundaries can cause neighbouring TADs to merge, creating ectopic contacts such as enhancer-promoter (E-P) loops that induce inappropriate gene activation. Examples include TAD border deletion leading to aberrant activation of LMNB1 and FOXG1. C. Mutations can prevent sub-TAD interactions such as E-P or active gene looping, abrogating gene activation. 
References

- of special interest

• of outstanding interest

1. Lodato S, Arlotta P: Generating neuronal diversity in the mammalian cerebral cortex. Annu Rev Cell Dev Biol 2015, 31:699-720.

2. Ofengeim D, Giagtzoglou N, Huh D, Zou C, Yuan J: Single-Cell RNA Sequencing: Unraveling the Brain One Cell at a Time. Trends Mol Med 2017, 23:563-576.

3. Williams RR, Azuara V, Perry P, Sauer S, Dvorkina M, Jorgensen H, Roix J, McQueen P, Misteli T, Merkenschlager $\mathrm{M}$, et al.: Neural induction promotes large-scale chromatin reorganisation of the Mash1 locus. J Cell Sci 2006, 119:132-140.

4. Martou G, Park PC, De Boni U: Intranuclear relocation of the PIc beta3 sequence in cerebellar purkinje neurons: temporal association with de novo expression during development. Chromosoma 2002, 110:542-549.

5. Peric-Hupkes D, Meuleman W, Pagie L, Bruggeman SW, Solovei I, Brugman W, Graf S, Flicek P, Kerkhoven RM, van Lohuizen M, et al.: Molecular maps of the reorganization of genomenuclear lamina interactions during differentiation. Mol Cell 2010, 38:603-613.

6. Walczak A, Szczepankiewicz AA, Ruszczycki B, Magalska A, Zamlynska K, Dzwonek J, Wilczek E, Zybura-Broda K, Rylski M, Malinowska M, et al.: Novel higher-order epigenetic regulation of the Bdnf gene upon seizures. J Neurosci 2013, 33:2507-2511.

7. Crepaldi L, Policarpi C, Coatti A, Sherlock WT, Jongbloets BC, Down TA, Riccio A: Binding of TFIIIC to sine elements controls the relocation of activity-dependent neuronal genes to transcription factories. PLoS Genet 2013, 9:e1003699.

8. Policarpi C, Crepaldi L, Brookes E, Nitarska J, French SM, Coatti A, Riccio A: Enhancer SINEs Link Pol III to Pol II Transcription in Neurons. Cell Rep 2017, 21:2879-2894.

9. Yanez-Cuna JO, van Steensel B: Genome-nuclear lamina interactions: from cell populations to single cells. Curr Opin Genet Dev 2017, 43:67-72.

10. Reddy KL, Zullo JM, Bertolino E, Singh H: Transcriptional repression mediated by repositioning of genes to the nuclear lamina. Nature 2008, 452:243-247.

11. Therizols P, Illingworth RS, Courilleau C, Boyle S, Wood AJ, Bickmore WA: Chromatin decondensation is sufficient to alter nuclear organization in embryonic stem cells. Science 2014, 346:1238-1242. 
12. Wittmann M, Queisser G, Eder A, Wiegert JS, Bengtson CP, Hellwig A, Wittum G, Bading H: Synaptic activity induces dramatic changes in the geometry of the cell nucleus: interplay between nuclear structure, histone H3 phosphorylation, and nuclear calcium signaling. J Neurosci 2009, 29:14687-14700.

13. Cebrian-Silla A, Alfaro-Cervello C, Herranz-Perez V, Kaneko N, Park DH, Sawamoto K, AlvarezBuylla A, Lim DA, Garcia-Verdugo JM: Unique Organization of the Nuclear Envelope in the Post-natal Quiescent Neural Stem Cells. Stem Cell Reports 2017, 9:203-216.

14. Kim Y, Sharov AA, McDole K, Cheng M, Hao H, Fan CM, Gaiano N, Ko MS, Zheng Y: Mouse Btype lamins are required for proper organogenesis but not by embryonic stem cells. Science 2011, 334:1706-1710.

15. Coffinier C, Jung HJ, Nobumori C, Chang S, Tu Y, Barnes RH, 2nd, Yoshinaga Y, de Jong PJ, Vergnes L, Reue K, et al.: Deficiencies in lamin B1 and lamin B2 cause neurodevelopmental defects and distinct nuclear shape abnormalities in neurons. $\mathrm{Mol}$ Biol Cell 2011, 22:4683-4693.

16. Giacomini C, Mahajani S, Ruffilli R, Marotta R, Gasparini L: Lamin B1 protein is required for dendrite development in primary mouse cortical neurons. Mol Biol Cell 2016, 27:35-47.

17. Gigante CM, Dibattista M, Dong FN, Zheng X, Yue S, Young SG, Reisert J, Zheng Y, Zhao H: Lamin B1 is required for mature neuron-specific gene expression during olfactory sensory neuron differentiation. Nat Commun 2017, 8:15098.

18. Robinson A, Partridge D, Malhas A, De Castro SC, Gustavsson P, Thompson DN, Vaux DJ, Copp AJ, Stanier P, Bassuk AG, et al.: Is LMNB1 a susceptibility gene for neural tube defects in humans? Birth Defects Res A Clin Mol Teratol 2013, 97:398-402.

19. Tatli M, Medalia O: Insight into the functional organization of nuclear lamins in health and disease. Curr Opin Cell Biol 2018, 54:72-79.

20. Nora EP, Lajoie BR, Schulz EG, Giorgetti L, Okamoto I, Servant N, Piolot T, van Berkum NL, Meisig J, Sedat J, et al.: Spatial partitioning of the regulatory landscape of the Xinactivation centre. Nature 2012, 485:381-385.

21. Dixon JR, Selvaraj S, Yue F, Kim A, Li Y, Shen Y, Hu M, Liu JS, Ren B: Topological domains in mammalian genomes identified by analysis of chromatin interactions. Nature 2012, 485:376-380.

22. Beagrie RA, Scialdone A, Schueler M, Kraemer DC, Chotalia M, Xie SQ, Barbieri M, de Santiago I, Lavitas LM, Branco MR, et al.: Complex multi-enhancer contacts captured by genome architecture mapping. Nature 2017, 543:519-524. 
23••. Bonev B, Mendelson Cohen N, Szabo Q, Fritsch L, Papadopoulos GL, Lubling Y, Xu X, Lv X, Hugnot JP, Tanay A, et al.: Multiscale 3D Genome Rewiring during Mouse Neural Development. Cell 2017, 171:557-572 e524.

In this thorough study, the authors map genome topology in incredibly high resolution throughout murine neuronal development, both in vitro and in vivo. Integrating the resultant maps with additional epigenetic data enables them to make some novel insights into genome organization during neuronal differentiation, as well as providing an important resource for the neuroscience community.

24•. Won H, de la Torre-Ubieta L, Stein JL, Parikshak NN, Huang J, Opland CK, Gandal MJ, Sutton GJ, Hormozdiari F, Lu D, et al.: Chromosome conformation elucidates regulatory relationships in developing human brain. Nature 2016, 538:523-527.

The authors generate high resolution maps of chromosome contacts during human corticogenesis, and use them to elucidate links between disease-risk SNPs and the sites they loop to.

25. Vian L, Pekowska A, Rao SSP, Kieffer-Kwon KR, Jung S, Baranello L, Huang SC, El Khattabi L, Dose $\mathrm{M}$, Pruett $\mathrm{N}$, et al.: The Energetics and Physiological Impact of Cohesin Extrusion. Cell 2018, 173:1165-1178 e1120.

26. Ong CT, Corces VG: CTCF: an architectural protein bridging genome topology and function. Nat Rev Genet 2014, 15:234-246.

27••. Nora EP, Goloborodko A, Valton AL, Gibcus JH, Uebersohn A, Abdennur N, Dekker J, Mirny LA, Bruneau BG: Targeted Degradation of CTCF Decouples Local Insulation of Chromosome Domains from Genomic Compartmentalization. Cell 2017, 169:930-944 e922.

Using auxin-inducible degradation to dramatically reduce CTCF levels, the authors examine the effect on genome conformation in ESCs, NPCs and postmitotic astrocytes, elegantly addressing the role of CTCF in genome organization.

28. Hirayama T, Tarusawa E, Yoshimura Y, Galjart N, Yagi T: CTCF is required for neural development and stochastic expression of clustered Pcdh genes in neurons. Cell Rep 2012, 2:345-357.

29•. Sams DS, Nardone S, Getselter D, Raz D, Tal M, Rayi PR, Kaphzan H, Hakim O, Elliott E: Neuronal CTCF Is Necessary for Basal and Experience-Dependent Gene Regulation, Memory Formation, and Genomic Structure of BDNF and Arc. Cell Rep 2016, 17:24182430. 
The authors demonstrate a critical role for CTCF in the genomic structure and activation of activity regulated genes, and in memory formation.

30. Elbert A, Vogt D, Watson LA, Levy M, Jiang Y, Brule E, Rowland ME, Rubenstein JLR, Berube NG: CTCF governs the identity and migration of MGE-derived cortical interneurons. $J$ Neurosci 2018.

31. Watson LA, Wang X, Elbert A, Kernohan KD, Galjart N, Berube NG: Dual effect of CTCF loss on neuroprogenitor differentiation and survival. J Neurosci 2014, 34:2860-2870.

32. Hadjur S, Williams LM, Ryan NK, Cobb BS, Sexton T, Fraser P, Fisher AG, Merkenschlager M: Cohesins form chromosomal cis-interactions at the developmentally regulated IFNG locus. Nature 2009, 460:410-413.

33. Sofueva S, Yaffe E, Chan WC, Georgopoulou D, Vietri Rudan M, Mira-Bontenbal H, Pollard SM, Schroth GP, Tanay A, Hadjur S: Cohesin-mediated interactions organize chromosomal domain architecture. EMBO J 2013, 32:3119-3129.

34. Fujita Y, Masuda K, Bando M, Nakato R, Katou Y, Tanaka T, Nakayama M, Takao K, Miyakawa T, Tanaka T, et al.: Decreased cohesin in the brain leads to defective synapse development and anxiety-related behavior. J Exp Med 2017, 214:1431-1452.

35. Kawauchi S, Calof AL, Santos R, Lopez-Burks ME, Young CM, Hoang MP, Chua A, Lao T, Lechner MS, Daniel JA, et al.: Multiple organ system defects and transcriptional dysregulation in the Nipbl(+/-) mouse, a model of Cornelia de Lange Syndrome. PLoS Genet 2009, 5:e1000650.

36. Boyle MI, Jespersgaard C, Brondum-Nielsen K, Bisgaard AM, Tumer Z: Cornelia de Lange syndrome. Clin Genet 2015, 88:1-12.

37. Uuskula-Reimand L, Hou H, Samavarchi-Tehrani P, Rudan MV, Liang M, Medina-Rivera A, Mohammed H, Schmidt D, Schwalie P, Young EJ, et al.: Topoisomerase II beta interacts with cohesin and CTCF at topological domain borders. Genome Biol 2016, 17:182.

38. Yang X, Li W, Prescott ED, Burden SJ, Wang JC: DNA topoisomerase Ilbeta and neural development. Science 2000, 287:131-134.

39. Madabhushi R, Gao F, Pfenning AR, Pan L, Yamakawa S, Seo J, Rueda R, Phan TX, Yamakawa H, Pao PC, et al.: Activity-Induced DNA Breaks Govern the Expression of Neuronal EarlyResponse Genes. Cell 2015, 161:1592-1605.

40. Davis JK, Broadie K: Multifarious Functions of the Fragile X Mental Retardation Protein. Trends Genet 2017, 33:703-714. 
41•. Sun JH, Zhou L, Emerson DJ, Phyo SA, Titus KR, Gong W, Gilgenast TG, Beagan JA, Davidson BL, Tassone F, et al.: Disease-Associated Short Tandem Repeats Co-localize with Chromatin Domain Boundaries. Cell 2018, 175:224-238 e215.

Research linking disease-causing tandem repeats, including those in FMR1, to sites that act as TAD and sub-TAD boundaries.

42. Giorgio E, Robyr D, Spielmann M, Ferrero E, Di Gregorio E, Imperiale D, Vaula G, Stamoulis G, Santoni F, Atzori C, et al.: A large genomic deletion leads to enhancer adoption by the lamin B1 gene: a second path to autosomal dominant adult-onset demyelinating leukodystrophy (ADLD). Hum Mol Genet 2015, 24:3143-3154.

43. Ibn-Salem J, Kohler S, Love MI, Chung HR, Huang N, Hurles ME, Haendel M, Washington NL, Smedley D, Mungall CJ, et al.: Deletions of chromosomal regulatory boundaries are associated with congenital disease. Genome Biol 2014, 15:423.

44. Danesin C, Houart C: A Fox stops the Wnt: implications for forebrain development and diseases. Curr Opin Genet Dev 2012, 22:323-330.

45. Ariani F, Hayek G, Rondinella D, Artuso R, Mencarelli MA, Spanhol-Rosseto A, Pollazzon M, Buoni S, Spiga O, Ricciardi S, et al.: FOXG1 is responsible for the congenital variant of Rett syndrome. Am J Hum Genet 2008, 83:89-93.

46. Mehrjouy MM, Fonseca ACS, Ehmke N, Paskulin G, Novelli A, Benedicenti F, Mencarelli MA, Renieri A, Busa T, Missirian C, et al.: Regulatory variants of FOXG1 in the context of its topological domain organisation. Eur J Hum Genet 2018, 26:186-196.

47. Ito K, Sanosaka T, Igarashi K, Ideta-Otsuka M, Aizawa A, Uosaki Y, Noguchi A, Arakawa H, Nakashima K, Takizawa T: Identification of genes associated with the astrocyte-specific gene Gfap during astrocyte differentiation. Sci Rep 2016, 6:23903.

48••. Monahan K, Horta A, Lomvardas S: LHX2- and LDB1-mediated trans interactions regulate olfactory receptor choice. Nature 2019, 565:448-453.

This exciting recent study demonstrates the importance of trans interactions of olfactory receptor genes and their enhancers in forming hubs that select one olfactory receptor gene for expression and silence all others.

49. Weintraub AS, Li CH, Zamudio AV, Sigova AA, Hannett NM, Day DS, Abraham BJ, Cohen MA, Nabet B, Buckley DL, et al.: YY1 Is a Structural Regulator of Enhancer-Promoter Loops. Cell 2017, 171:1573-1588 e1528. 
50•. Beagan JA, Duong MT, Titus KR, Zhou L, Cao Z, Ma J, Lachanski CV, Gillis DR, Phillips-Cremins JE: YY1 and CTCF orchestrate a 3D chromatin looping switch during early neural lineage commitment. Genome Res 2017, 27:1139-1152.

This study demonstrates a novel role for YY1 in chromosome looping in neuronal cells, and explores the decreasing role of CTCF during neuronal differentiation.

51. Tang Z, Luo OJ, Li X, Zheng M, Zhu JJ, Szalaj P, Trzaskoma P, Magalska A, Wlodarczyk J, Ruszczycki B, et al.: CTCF-Mediated Human 3D Genome Architecture Reveals Chromatin Topology for Transcription. Cell 2015, 163:1611-1627.

52. Fan H, Lv P, Huo X, Wu J, Wang Q, Cheng L, Liu Y, Tang QQ, Zhang L, Zhang F, et al.: The nuclear matrix protein HNRNPU maintains 3D genome architecture globally in mouse hepatocytes. Genome Res 2018, 28:192-202.

53. Barutcu AR, Lajoie BR, Fritz AJ, McCord RP, Nickerson JA, van Wijnen AJ, Lian JB, Stein JL, Dekker J, Stein GS, et al.: SMARCA4 regulates gene expression and higher-order chromatin structure in proliferating mammary epithelial cells. Genome Res 2016, 26:1188-1201.

54. Ito K, Noguchi A, Uosaki Y, Taga T, Arakawa H, Takizawa T: Gfap and Osmr regulation by BRG1 and STAT3 via interchromosomal gene clustering in astrocytes. Mol Biol Cell 2018, 29:209-219.

55. Kagey MH, Newman JJ, Bilodeau S, Zhan Y, Orlando DA, van Berkum NL, Ebmeier CC, Goossens J, Rahl PB, Levine SS, et al.: Mediator and cohesin connect gene expression and chromatin architecture. Nature 2010, 467:430-435.

56. He Y, Dupree J, Wang J, Sandoval J, Li J, Liu H, Shi Y, Nave KA, Casaccia-Bonnefil P: The transcription factor Yin Yang 1 is essential for oligodendrocyte progenitor differentiation. Neuron 2007, 55:217-230.

57. Gabriele M, Vulto-van Silfhout AT, Germain PL, Vitriolo A, Kumar R, Douglas E, Haan E, Kosaki K, Takenouchi T, Rauch A, et al.: YY1 Haploinsufficiency Causes an Intellectual Disability Syndrome Featuring Transcriptional and Chromatin Dysfunction. Am J Hum Genet 2017, 100:907-925.

58. Roussos P, Mitchell AC, Voloudakis G, Fullard JF, Pothula VM, Tsang J, Stahl EA, Georgakopoulos A, Ruderfer DM, Charney A, et al.: A role for noncoding variation in schizophrenia. Cell Rep 2014, 9:1417-1429. 
59. Gallagher MD, Posavi M, Huang P, Unger TL, Berlyand Y, Gruenewald AL, Chesi A, Manduchi E, Wells AD, Grant SFA, et al.: A Dementia-Associated Risk Variant near TMEM106B Alters Chromatin Architecture and Gene Expression. Am J Hum Genet 2017, 101:643-663.

60. Bharadwaj R, Jiang Y, Mao W, Jakovcevski M, Dincer A, Krueger W, Garbett K, Whittle C, Tushir JS, Liu J, et al.: Conserved chromosome $2 q 31$ conformations are associated with transcriptional regulation of GAD1 GABA synthesis enzyme and altered in prefrontal cortex of subjects with schizophrenia. J Neurosci 2013, 33:11839-11851.

61. Amendola M, van Steensel B: Nuclear lamins are not required for lamina-associated domain organization in mouse embryonic stem cells. EMBO Rep 2015, 16:610-617.

62. Ibarra A, Hetzer MW: Nuclear pore proteins and the control of genome functions. Genes Dev 2015, 29:337-349.

63. Toda T, Hsu JY, Linker SB, Hu L, Schafer ST, Mertens J, Jacinto FV, Hetzer MW, Gage FH: Nup153 Interacts with Sox2 to Enable Bimodal Gene Regulation and Maintenance of Neural Progenitor Cells. Cell Stem Cell 2017, 21:618-634 e617. 
Figure. 1
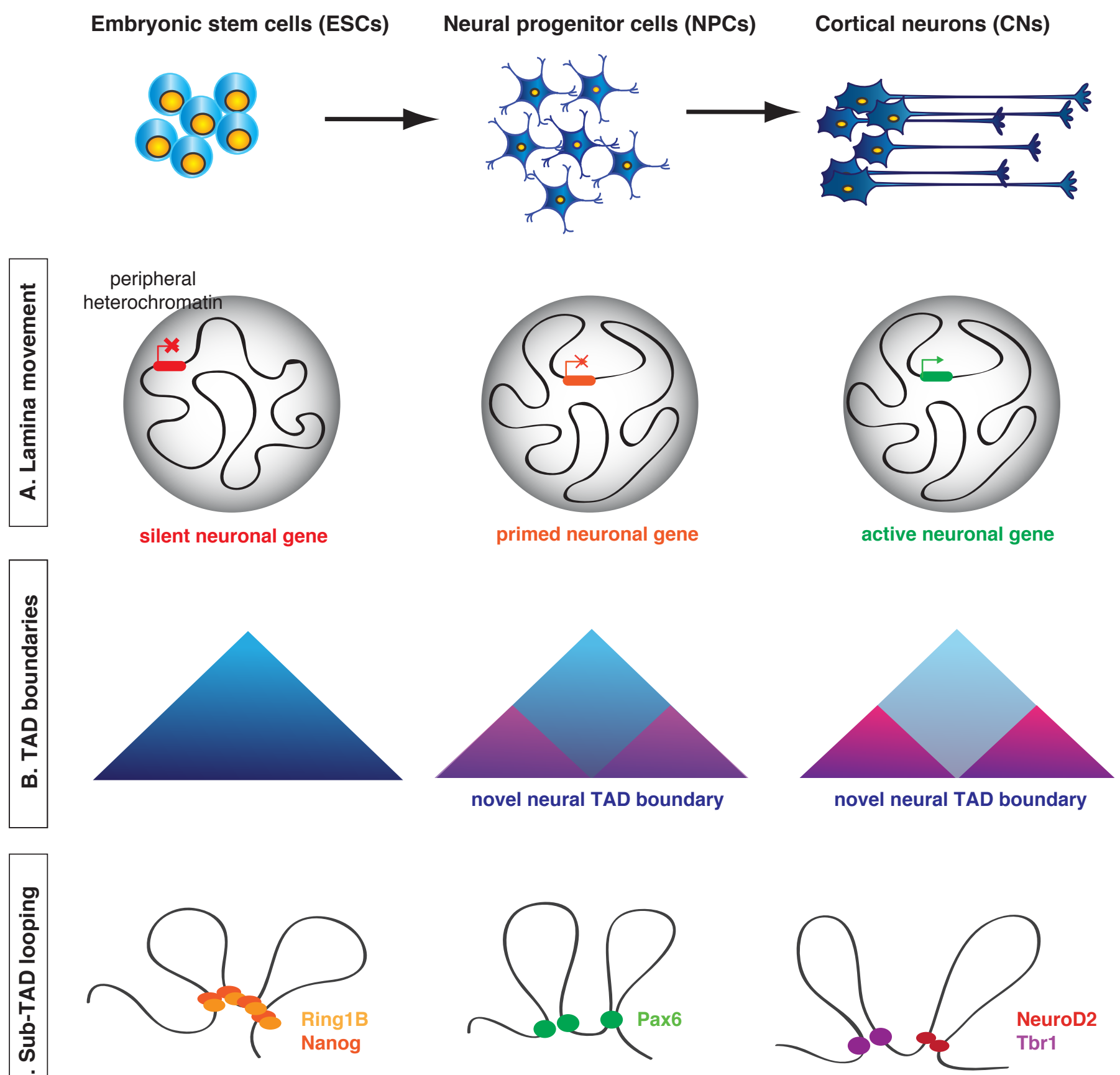
Figure. 2

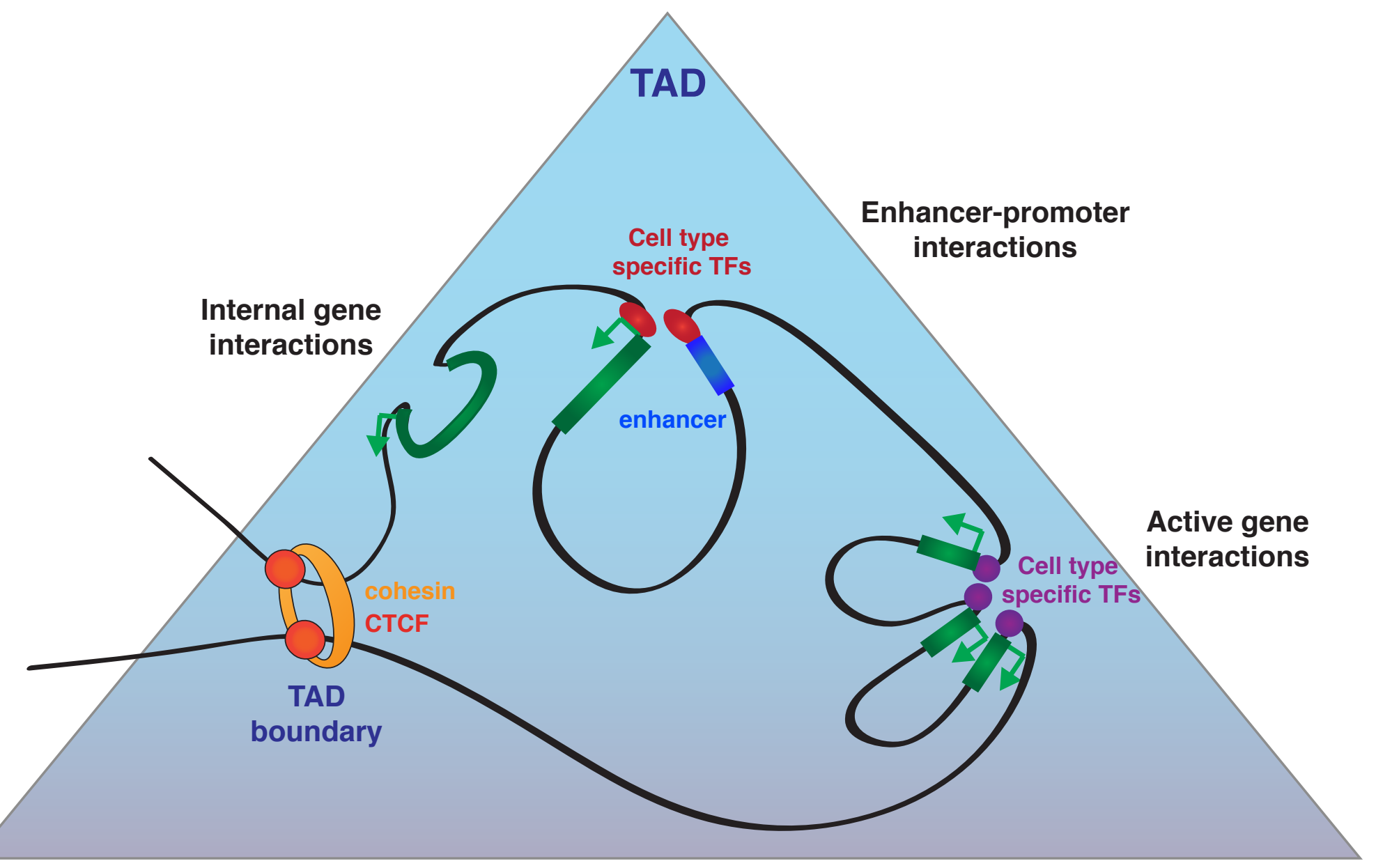


Figure 3

\section{(a) Organizer gene mutation}

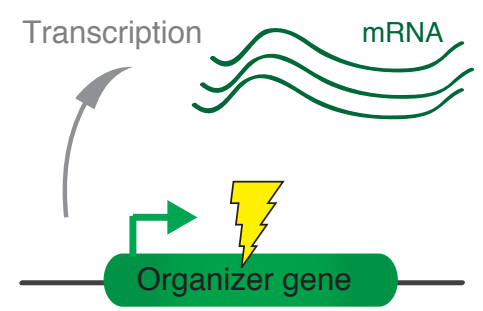

Loss of

structural protein

Aberrant looping,

transcriptional dysregulation

Organizer gene

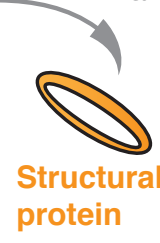
protein

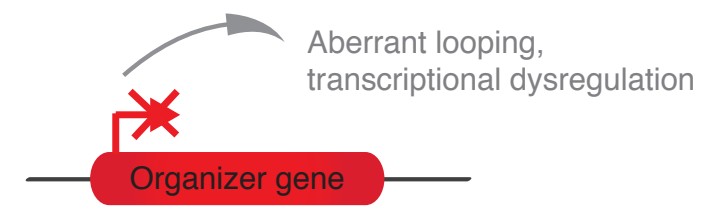

\section{(b) TAD boundary mutation} Translation
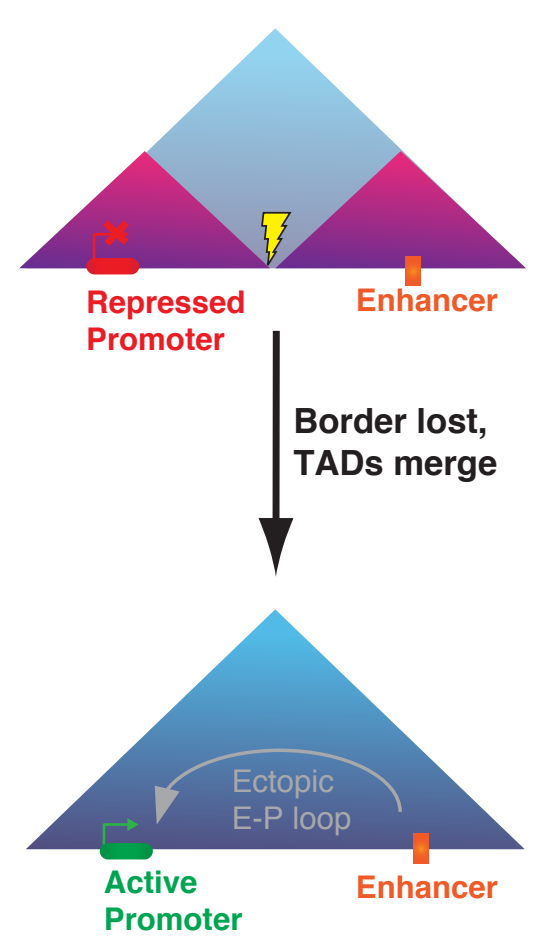

Border lost, TADs merge

\section{Promoter}

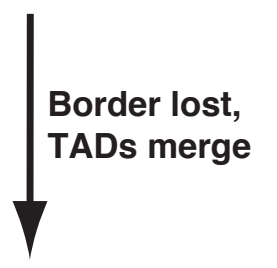

Promoter (c) Enhancer mutation

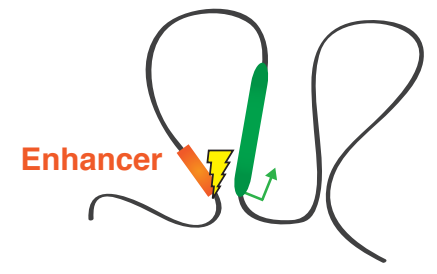

Loss of E-P loop

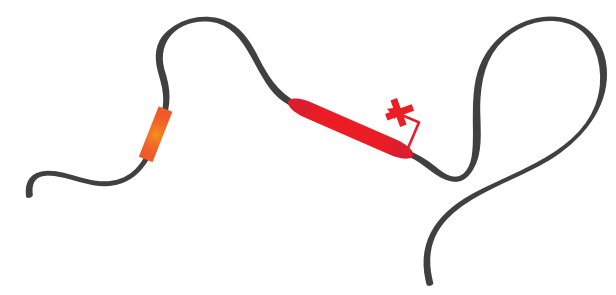

